\title{
LAS ELECIONES EN LA FRONTERA MÉXICO-ESTADOS UNIDOS. REVISANDO LAS TENDENCIAS
}

\author{
Por \\ Samuel Schmidt*
}

\begin{abstract}
RESUMEN
En este articulo se revisan las tendencias electorales en México de 1964 a 1988 y se comparan con las tendencias electorales registradas en el mismo periodo en los estados mexicanos que hacen frontera con los Estados Unidos.

Tres tendencias son muy claras: dismimución de la votación a favor del PRL, aumento de la votación a favor del paN y aumento de la abstención. Aun cuando en algunos casos en algunos estados la tendencia no es muy clara, no existe ningún elemento que haga ver la existencia de un comportamiento electoral en la frontera distinto al registrado en el resto del país.

En la 6ptica anterior se hace una revisión rápida de las elecciones en 1989. incluido el triunfo panista en Baja California.
\end{abstract}

\begin{abstract}
A review of the electoral trend in Mexican states bordering the United States between 1964 and 1988 is done in this article.

Three trends are very clear: decrease in the PRI vote, increase in the PAN vote and increase in abstention. Eventhough in some cases in some states the trend is not very clear, there is not an element to show that the border electoral behavior is different than the electoral behavior in the rest of the country.

Under this perspective the author makes a quick revision of the 1989 elections including the PAN victory in the Baja California governor election.
\end{abstract}

Se ha vuelto común decir que las elecciones del 6 de julio de 1988 fueron un parteaguas, sin embargo, aparte del hecho de que se haya registrado una escisión en el PRI, las tendencias electorales que se daban en el país en los últimos veinte affos continuaron. De esta manera, la elección de 1988 no representa una modificación del comportamiento electoral, aunque sugiere la confirmación de ciertas hipótesis sobre el comportamiento electoral mexicano.

- Profesor asociado en la Facultad de Ciencias Políticas y Sociales, unAM y Profesor visitante en San Diego State University. 


\section{PARTIDOS POLÍTICOS Y CLIENTELA ELECTORAL}

Anthony Downs (1973) sostiene que el voto es un valor económico; por ello, los ciudadanos asumen que van a invertir su valor ahí donde van a tener una mayor ganancia: Como consecuencia de esto encuentra que los partidos políticos se dirigen hacia el espacio político-ideológico donde se localizan la mayoría de los votantes, esto es, en el centro.

A partir de 1970 se inicia en México el surgimiento acelerado de partidos políticos, que mostró, además de los cuatro partidos con registro electoral (PAN, PARM, PPS, PRI) y del no registrado partido comunista en los setenta, un serio incremento para los ochenta donde surgieron el PMT, PST, PRT, PPM, PSD y PDM junto con una gran cantidad de movimientos políticos. Algunos de estos partidos se escindieron de otros partidos políticos como el PPM, que se escindió del PPS ${ }^{1}$, otros fueron el resultado de fusiones de pequeños grupos políticos, como es el caso de los grupos trotskistas que dieron lugar al PRT ${ }^{2}$, otros son partidos de nueva formación como son el PMT y PST.

Para las elecciones de 1988 la izquierda vivió un proceso de fusión, llevando el número de partidos contendientes en las elecciones a ocho (PAN, PARM, PDM, PFCRN ${ }^{3}$, PPS, PMS, PRI, PRT) que en realidad, y debido a la formación del Frente Democrático Nacional $\left(\mathrm{FDN}^{4}\right)$, fueron solamente cinco (PAN, FDN, PDM, PRI, PRT).

Si bien el nuevo marco partidista nacional cubría prácticamente todo el espectro ideológico, se notó un fuerte acercamiento hacia el centro de casi todos los partidos. Los partidos de izquierda eliminaron la palabra socialista de sus nombres y el PRI y el PAN llegaron a coincidir sorprenden-

1 Las escisiones son lo que se podría decir movimientos naturales en el proceso de madurez de los partidos políticos. En 1988 una escisión en el PRI dio lugar al Frente Democrático Nacional y posteriormente al Partido de la Revolución Democrática.

2 El caso de la formación del PRT fue notorio porque no era común en México ver un proceso de fusión dentro de la izquierda, pero ya para 1988 este partido sufrió una escisión cuando se formó el Movimiento al Socialismo que de inmediato se alió al Frente Democrático Nacional. 3 La trayectoria del PFCRN es por demás aleccionadora sobre cómo los partidos políticos mexicanos pueden gravitar alrededor del gobiemo. En los setenta un grupo se escinde del Comite Nacional de Auscultación y Organización (CNAO) lidereado por Heberto Castillo y forman el Partido Socialista de los Trabajadores (PST) apoyado por la presidencia de la república y diversos funcionarios gubemamentales. Posteriormente recibe apoyo del sindicato de petroleros y para 1988 se plantea "un cambio de piel" y asume una identidad cardenista cambiando su nombre por el de Partido del Frente Cardenista de Reconstrucción Nacional (PFCRN), confundiéndo a la opinión pública sobre si era el partido de Cárdenas o no, lo que provocó una escisión en el partido con un grupo que se fue al Partido Mexicano Socialista. En 1988 ambos grupos se encontraron en el FDN. El PFCRN fue el primer partido que apoyó al gobiemo de Salinas para romper con el bloque de izquierda. Los datos sobre los apoyos gubemamentales al PST los consiguió el autor en entrevistas a miembros de ese partido.

4 El FDN contenía al PARM, PRCRN, PPS, PMS y a un grupo escindido del PRI denominado la Corriente Democrática. 
temente en sus propuestas. Los partidos que se quedaron en los extremos tuvieron una votación tan reducida -el PRT el $1.04 \%$ y el PDM el $0.42 \%$ - que tuvieron que desaparecer legalmente, ya que la ley electoral establecía que aquel partido que no alcanzara el $1.5 \%$ de la votación perdía su registro electoral. Este indicador parece mostrar que el electorado rechaza los extremos.

Es así como se puede considerar que los partidos políticos, cuya distribución ideológica aproximada se presenta en la figura 1, pelearon la porción de los votos que se encuentra en el centro del espectro, lo que hace pensar que el supuesto de Downs, en el sentido que es ahí donde en realidad se encuentra la mayor parte de los votantes, es correcto.

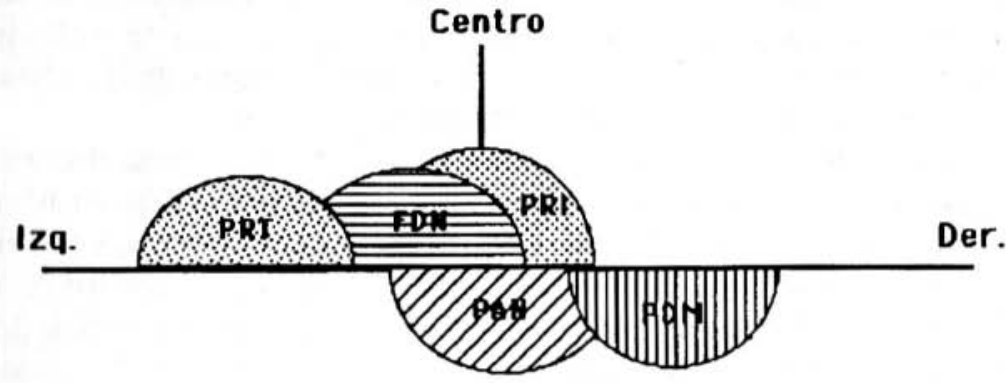

FIGURA 1. Ubicación político-ideológica de los partidos políticos mexicanos.

NOTA: El PDN incluye al PMS, PARM, PPS, PMT, PFCRN. La representación gráfica no equivale al peso electoral de cada partido.

Las porciones electorales que logró cada partido en 1988 para la elección presidencial, como se ve en el cuadro 1 , modifican en cierta medida la tendencia electoral, situación que se puede explicar por varias razones: 1) porque el surgimiento de una oposición priísta fuera del PRI aceleró la caída de la votación por el PRI y trasladó esos votos hacia partidos títeres del PRI en el pasado (PARM, PPS, PFCRN); 2) hipotéticamente esto se debe al hecho que los diversos partidos políticos se acercaron hacia el centro, llegando algunos de ellos inclusive a competir con algunos de los símbolos priístas o con símbolos entronizados por el PRI, como es la imagen de Lázaro Cárdenas. Por un lado, el PST cambió su nombre al de Partido del Frente Cardenista de Reconstrucción Nacional y la

5 La polémica que sostuvo el PRD con el PRI en 1989, respecto a su derecho a utilizar los colores de la bandera en el escudo partidista, puede mostrar cómo la pelea electoral se encuentra dirigida hacia una porción fija de la población. 
coalición de izquierda —el FDN- postuló como su candidato presidencial al hijo de Lázaro Cárdenas. ${ }^{6}$

Tal vez el caso más notorio de desplazamiento hacia el centro de algunos de los partidos políticos sea el del Partido Comunista, que empieza a transformarse por medio de fusiones con otros partidos y grupos. Primero se convirtió en Partido Socialista Unificado de México, eliminando la palabra comunista de su nombre, pasa después a Partido Mexicano Socialista, postulando en 1988 como candidato a la presidencia a un izquierdista no marxista (Heberto Castillo). Este último declina su candidatura en favor de un expriísta (Cuauhtémoc Cárdenas). El partido llega a las elecciones de 1988 como parte de un Frente Democrático, borrando la nomenclatura socialista de su nombre. De esa manera, el PMS, como parte de una gran coalición de izquierda, buscó - y en gran medida lo logró- ponerse en aproximadamente el mismo espacio político-ideológico que el PRI.

Esta "centrización" de los partidos políticos altero la tendencia electoral, llamando la atención casos como el del PARM —que de 1982 a 1988 amplió su porción de los votos en $418 \%$ - el del PFCRN —que aumentó sus votos en $750.7 \%$ - y que la suma de la votación de los partidos miembros de la coalición del FDN aumentó en un $390.9 \%$. Por lo que toca a la pérdida del PRI, este partido cargó con el trauma de Tlatelolco y perdió el $5.1 \%$ de los votos de 1964 a 1970; asimismo con el fin conflictivo del periodo presidencial de Luis Echeverría perdió el $1.2 \%$ de 1970 a 1976, aun con la caída de la economía, devaluación y expropiación de los bancos de José López Portillo cayó en $21.2 \%$ de 1976 a 1982; de 1982 a 1988 de nuevo disminuyó su votación en $22.5 \%$. Si bien la pérdida de votos a favor del PRI fue durante cuatro elecciones, la caída fue moderada hasta 1976, cuando en dos elecciones consecutivas se nota una caída drástica que se puede explicar porque ya había una oferta de partidos políticos que ofrecían alternativas, aunque habría que decir que la "centrización" de los partidos políticos en 1988 planteaba propuestas a la población sin grandes conflictos ideológicos, ${ }^{7} \mathrm{de}$

6 En plática de este autor con Heberto Castillo, candidato presidencial del PMS electo en elecciones primarias, señaló que uno de los criterios para declinar su candidatura a favor de Cuauhtémoc Cárdenas fue el apellido. Creo, sin embargo, que no hay que menospreciar la relación que Castillo tuvo con Lázaro Cárdenas. Hay que mencionar también que el gobiemo mexicano preparo a 1988 como un año netamente Cardenista. Ese año era el cincuentenario de la expropiación petrolera, aunque también se destacaron otros grandes proyectos cardenistas, aspecto que la oposición, en especial el FDN, aprovechó muy bien. En especial porque ellos eran los que tenían como candidato al hijo del general.

7 Ver las posturas de los diferentes partidos en Dávila, Enrique, "Plataformas electorales e índice analítico"; Meyenberg, Yolanda, et al., Política y partidos en las elecciones federales de 1985, México, UNAM, Facultad de Ciencias Políticas y Sociales, 1987. Si bien este estudio fue hecho para las elecciones de 1985, se puede considerar válido para las de 1988. 
ahf que simplemente reconocer la existencia de estos partidos y opciones no nos ayuda a explicar el declive histórico de la votación del PRI, cuestión que por sí misma está más allá de los objetivos de este artículo.

El partido que rompe el esquema es el PAN, ya que se puede considerar que se ubica ideológicamente más o menos en el mismo ámbito que el PRI y sin embargo su aumento en la captación de votos de 1982 a 1988 fue de un módico $8.72 \%$.

CUADRO 1. Votación en la elección presidencial, 1964-1988. Porcentajes.

\begin{tabular}{lccccc}
\hline Partido/Afio & 1964 & 1970 & 1976 & 1982 & 1988 \\
\hline PRI & 87.8 & 83.3 & 82.3 & 65.0 & 50.4 \\
PAN & 10.9 & 13.8 & $\mathrm{a}$ & 15.7 & 17.1 \\
PDN* & 1.2 & 1.4 & 6.8 & 7.9 & 30.9 \\
PPS & 0.7 & 0.9 & 3.7 & 1.5 & 10.5 \\
PFCRN & $\mathrm{b}$ & $\mathrm{b}$ & $\mathrm{b}$ & 1.4 & 10.5 \\
PARM & 0.5 & 0.5 & 3.1 & 1.5 & 6.3 \\
PMS & $\mathrm{c}$ & $\mathrm{c}$ & $\mathrm{c}$ & 3.5 & 3.6 \\
PDM & $\mathrm{d}$ & $\mathrm{d}$ & $\mathrm{d}$ & $\mathrm{d}$ & 1.0 \\
PRT & $\mathrm{d}$ & $\mathrm{d}$ & $\mathrm{d}$ & 1.8 & 0.4 \\
PSD & $\mathrm{e}$ & $\mathrm{e}$ & $\mathrm{e}$ & 0.2 & $\mathrm{e}$ \\
& & & & & \\
\hline
\end{tabular}

MUINTB: Para 1988, Molinar Horcasitas, Juan, "Crónica del día en que el sistema se cayó-calló", El Cotidiano, no. 25, (México, 1988). Para 1964 a 1982, Schmidt, Samuel, "Votación y elecciones en la frontera México-Estados Unidos, 1961-1982.". En Schmidt, S., J.W., Wilkie, M., Esparza, M. (comp.), "Estudios Cuantitativos sobre la historia de México", (México, UNAM, 1988).

* Hasta 1982, para mostrar la evolución en su votación, se presenta la suma de votos que hubieran tenido todos los partidos del frente si acaso se hubieran coalicionado (PPS, PFCRN, PARM, PMS).

a. No presentó candidato presidencial.

b. Este partido no existía en 1982, logró votos como Partido Socialista de los Trabajadores, para 1988 cambió de nombre.

c. Era el Partido Comunista que se encontraba en la "ilegalidad", o sea que no estaba reconocido por el gobierno. En 1982 logró votos como Partido Socialista Unificado de México (PSUM).

d. No existía.

c. Este partido no existía antes de 1982 y perdió su registro electoral en 1982 por no lograr la votación mínima que establece la ley. 


\section{PARTIDOS POLÍTICOS Y ABSTENCIÓN ELECTORAL}

La presencia de una mayor cantidad de partidos políticos supone una mayor oferta de opciones político-ideológicas, de tal manera que la mayor parte de la sociedad cuenta con un menú político suficiente para satisfacer sus diversos intereses. Lo paradójico del caso mexicano es que aun cuando la oferta de partidos políticos aumenta, también lo hace la abstención. En este sentido la elección de 1988 no es ningún parteaguas, sino que, como se ve en el cuadro 2 , este evento registra la continuación de la misma tendencia electoral. ${ }^{8}$

Los datos del cuadro 2 sugieren la existencia de una posible manipulación, puesto que es difícil aceptar las variaciones que la abstención registra, tanto cuando aumenta como cuando disminuye, pero todavía más sorprendente es la diferencia que registran las distintas fuentes, como por ejemplo en 1976, donde la fuente 1 registra el $38.1 \%$, la 2 el $54.6 \%$ y la 3 el $63.5 \%$. En otro lado (Schmidt, 1988) he mostrado que en elecciones legislativas, como la de 1979 , la abstención fue del $80.6 \%$ mientras que en 1973 la misma fuente reporta un $52.4 \%$ de abstención, pero no queda claro en base a qué causas hay un incremento del 53.8 en la abstención en sólo seis afios.

Por otro lado, desde 1958 tenemos que, la presencia legislativa de la oposición ha ido en aumento. En ese affo la oposición aparece con los diputados de partido y va incrementando su presencia hasta el grado que, para 1988, controla el $48 \%$ de las curules de la Cámara de Diputados y cuatro de las sesenta y cuatro senadurías. Sin embargo, aun cuando esta presencia política demuestra que hay mayor opción de selección para el ciudadano, la abstención no se reduce y en cambio aumenta, sugiriendo que los partidos políticos pelean por la misma porción de los votos y que esta porción no se incrementa en términos porcentuales.

Dado que la mayoría de la población no vota, la abstención es entonces un componente fundamental para entender el comportamiento político de los individuos. De ahí podemos tener elementos para inferir la percepción del ciudadano sobre el sistema político, ya que si las elecciones son el

\footnotetext{
8 Siempre es aconsejable tener una actitud precautoria sobre las cifras mexicanas y su manipulación estadística, ya que de no hacerlo se puede poner en entredicho cualquier acercamiento teórico. Por ejemplo, para considerar la abstención electoral, dos fuentes distintas calculan la población en edad de votar con una gran diferencia, la primera, Equipo de Coyuntura, "De la transición democrática", El Cotidiano, no. 25, (México, 1988) la calcula en 52'207,600 y López, Arturo, Geografia de las elecciones presidenciales de México, 1988. Fundación Arturo Rosenblueth, México, la calcula en 41'893,656, con una diferencia entre ambas de $19.75 \%$. La solución que este autor ha encontrado consiste en estudiar las tendencias y agregar al estudio de las cifras un análisis cualitativo para tener una mejor aproximación.
} 
CUADRO 2. Abstención total en elecciones presidenciales en México, 1964-1988² (porcentajes).

\begin{tabular}{cccc}
\hline Año & Fuente 1 & Fuente 2 & Fuente $3^{\mathrm{b}}$ \\
\hline 1964 & 33.3 & 38.9 & $\mathrm{c}$ \\
1970 & 35.0 & 41.4 & 57.6 \\
1976 & 38.1 & 54.6 & 63.5 \\
1982 & 28.5 & 43.2 & $\mathrm{c}$ \\
1988 & $\mathrm{c}$ & 63.3 & $\mathrm{c}$ \\
\hline
\end{tabular}

FUINTE 1: Excelsior, 16 de julio, 1982.

2: De 1964 a 1982, cálculo del autor a partir de Reforma Política, Secretaría de Gobernación, Comisión Federal Electoral, México, 1982. Para 1988 cálculo del autor a partir de "De la transición democrática", $E l$ Cotidiano, no. 25, sept-oct. 1988.

3: Paoli, José, "Primer fracaso de la reforma política", Proceso, no. 142, México, 1979.

a. Por abstención total se considera: abstención al empadronamiento, más abstención al voto, más votos anulados. Para 1988 en los reportes de votación esta última categoría ha desaparecido, así que para este año esta cifra total de abstención en realidad es mayor.

b. El análisis de este autor cubre de 1970 a 1979.

c. No lo cubre la fuente.

instrumento para medir el consenso y uno de los instrumentos para construir la legitimidad, el hecho de que aumente el número de ciudadanos que no participan, ya sea porque no tienen el conocimiento necesario para participar electoralmente, porque no tienen interés en la participación electoral, porque no se satisfacen con ninguna de las opciones partidistas o se oponen a todas las opciones partidistas existentes, muestra que esta mayoria está expresando una opinión de rechazo a las opciones que le presenta el sistema político en general.

También existe la posibilidad de que los ciudadanos decidan participar más activamente en un cierto tipo de elección que en otro, y emitan más votos en aquellas elecciones con las que sientan que pueden influir más. En este sentido, tenemos que las elecciones presidenciales, posiblemente por la imagen de omnipotencia del presidente mexicano, registran menor abstención que las elecciones para diputados y algunas elecciones para diputados locales, presidentes municipales y gobernador, que si bien supuestamente tienen una influencia directa sobre la vida de los ciudadanos, los 
electos tienen menor poder ${ }^{9}$. Estas últimas elecciones llegan a registrar una abstención muy por encima de la registrada en las elecciones presidenciales. $^{10}$

Si bien debemos reconocer la ausencia de análisis sobre la abstención electoral, tal vez sea ahora el momento en que empecemos a reflexionar sobre ella, y que por lo menos planteemos varias hipótesis para explicar no solamente su existencia, sino que también preguntemos por qué dentro del comportamiento electoral es el rubro que mayor cantidad de "votos" recibe. En este contexto vale la pena plantear viarias hipótesis:

1) La sociedad mexicana en realidad no está preocupada por votar ya que no piensa que es en el terreno electoral donde se establecen las modificaciones sociales, económicas y políticas más significativas, por lo tanto, las elecciones le son totalmente irrelevantes.

2) En torno a la abstención, la sociedad está dividida en varios sectores que se encuentran en todas las clases sociales:

a) El sector apático, el cual está desmotivado para votar.

b) El sector ignorante, que no tiene información ni sabe cuál es el valor político y legal de las elecciones, para este sector la legitimidad es un valor inexistente.

c) El sector opositor, que se opone al sistema político-electoral, así su abstención representa: rechazo a todos los partidos políticos, o bien, rechazo al sistema político en general y en su totalidad.

3) La abstención es un rechazo al eurocentrismo, visto éste como la imposición cultural, económica y social proveniente de la dominación europea, que se inició con la conquista y colonización europea, y que los mexicanos aún resienten. Una forma de protesta sería el rechazo a la forma partido político como medio de representación, en virtud de que también es un invento curopeo.

4) Como ya se dijo, en virtud de que las acciones (favores, castigos y recompensas) del gobierno mexicano se definen a partir de diversas

9 Este tipo de análisis debe llegar hasta el nivel municipal en un periodo histórico tan amplio como lo permitan las fuentes.

10 En algunos de los reportes preliminares de las elecciones de 1989 en Baja California, se menciona que la abstención fue entre el 64 y el 66\%, Excélsior. (México, D.F.; julio 11, 1988). "en los comicios federales de 1988 acudieron más votantes que en los comicios de la semana pasada..., esos vaivenes de la voluntad se deben a que no hay una firme cultura política" declaró Genamo Alamilla presidente de la Comisión Episcopal de Pastoral Social, El Financiero, (México D.F.: julio 11, 1989). En Campeche la abstención fue entre 62 y $66 \%$ mientras que en Durango fue de 75\%, Excélsior (México D.F; julio 13, 1989). El Financiero (julio 13, 1989) discrepa respecto a Durango y establece la abstención en un 70\%. Proceso (julio 17, 1989) por su parte la calcula en $89 \%$, aunque aquí se le ubica de la siguiente manera: "Era de esperarse que con dos fraudes seguidos (1986 y 1988) el pueblo duranguense desairara las elecciones" según declaró el diputado panista Rodolfo Elizondo, p. 8. 
presiones, es en el terreno de la organización donde se articulan las fuerzas políticas para presionar, por lo tanto, las votaciones son totalmente irrelevantes. Desde este punto de vista, la legitimidad y el consenso no se establecen por medio de las votaciones, sino por medio de los apoyos, lealtad y disciplina que se le otorgan al gobierno.

Si bien me inclino a pensar que esta última es una hipótesis muy sugerente y de peso, también creo que es el reflejo de una situación que está cambiando con rapidez, y que si bien retrata al viejo corporativismo mexicano, puede ser que también sea uno de sus últimos rasgos.

\section{LAS VOTACIONES EN LA FRONTERA MÉXICO-ESTADOS UNIDOS}

El análisis de las elecciones en la frontera México-Estados Unidos hipotéticamente podría sugerir la existencia de un patrón distinto al patrón nacional, dada la supuesta o posible influencia de Estados Unidos o al posible hecho de que sea una región significativamente distinta al resto del país; sin embargo esto no es así.

En primer lugar, creo que la imagen de la influencia estadounidense es una recreación intelectual, tal vez un poco desviada de la realidad. Se considera que Estados Unidos en comparación con México sí es una verdadera democracia - lo cual puede discutirse-, pero se ignora que en las elecciones de 1988 en Estados Unidos la abstención llegó al 50\%, esto es menor a la mexicana, aunque no lo fuera así en años anteriores. ${ }^{11}$

Por otro lado, en Estados Unidos parece existir la imagen de que las elecciones son un medio de equilibrio de poderes en el marco federal y, tal como se mencionó, para México hasta el momento eso parece ser irrelevante. Así, el factor democrático en Estados Unidos consiste en la existencia de una posibilidad de representación del pueblo en el congreso y de competencias abiertas que se manejan con honestidad para balancear a los poderes. En México, hoy en día, por democracia parece ser que se ha llegado a entender estrictamente la derrota del PRI, lo cual puede ser correcto pero solamente en el sentido de que tal derrota implique a su vez la eliminación de las prácticas autoritarias en el sistema político mexicano.

Adicionalmente, no se dispone de ningún elemento para sostener el argumento de que el comportamiento electoral en la frontera norte de

11 Ver ambas tendencias en una serie de más de 100 años en J. Wilkie y D. Lorey (eds.) Statistical Abstract of Latin America, vol. 25 Los Angeles, UCLA: Latin American Center Publications, 1987 pp. 820 y 900. 
México sea sustancialmente distinto al del resto del pars, aunque podemos considerar que el rechazo al "centro"12 que históricamente ha habido, puede explicar el porqué en esa región se ha registrado un fuerte voto de oposición, lo que nos llevaría a discutir si el voto a favor de Cuauhtémoc Cárdenas en Baja California en 1988 se debió a que a él se le consideró como anti-centro, o si en dichas elecciones hubo también una cierta consideración ideológica o de rechazo al PRI.

Por otro lado, se menciona que las características específicas de la frontera son sustancialmente diferentes a las del resto del país, en especial por lo que se refiere a las condiciones socioeconómicas, lo cual parece ser cierto. Como se puede ver en el cuadro 3, en efecto, la frontera norte presenta algunos cambios interesantes respecto a los indicadores nacionales.

\section{CUADRO 3. Indicadores de población de seis estados fronterizos, 1980, (porcentajes).}

\begin{tabular}{|c|c|c|c|c|c|c|c|}
\hline PEA & Nal. & B. C. & Son. & Chih. & Coah. & $\begin{array}{l}\text { Nvo. } \\
\text { León }\end{array}$ & $\begin{array}{c}\text { Tamau- } \\
\text { lipas }\end{array}$ \\
\hline Agrícola & 25.8 & 9.5 & 10.1 & 20.7 & 15.8 & 8.4 & 18.0 \\
\hline Manuf. & 11.7 & 13.6 & 4.1 & 12.4 & 14.4 & 24.6 & 11.9 \\
\hline Pob. Urbana & 66.3 & 85.3 & 70.5 & 70.3 & 75.8 & 87.4 & 75.1 \\
\hline Educación* & 25.3 & 34.5 & 31.1 & 25.2 & 29.2 & 38.1 & 28.0 \\
\hline Nac. en otro Edo. & 47.1 & 43.7 & 16.3 & 12.2 & 15.2 & 24.7 & 23.4 \\
\hline
\end{tabular}

FUENTE: James T. Peach, “Demographic and economic change in Mexico's northem frontier: Evidence from the $x$ Censo General de Población y Vivienda", Latin American Research Papers, no. 3 (Las Cruces, New Mexico State University, Center for Latin American Studies, 1984).

* Población con doce años o más de educación.

12 Es muy interesante notar que, cuando se supo la noticia del triunfo de Emesto Ruffo (PAN) a la gubernatura de Baja California en 1989, los prístas protestaron contra el centro gritando "-Fuera chilangos I- INo queremos ayuda del centrol" cuando en realidad de los enviados del centro no había ningún "chilango" o ciudadano del D.F. Los enviados fueron Luis H. Ducoing ex-gobernador de Guanajuato, Ismael Orozco (Jalisco), Eduardo Robledo (Chiapas), Manuel Ramos Gurrión (Veracruz), Carlos Ortiz Tejeda (Coahuila) y el coordinador de la campaña prísta que fue allegado de Cuauhtémoc Cárdenas en Michoaćn. Esto me hace pensar que el rechazo al PRI es porque representa la imagen del centro y en este caso, el PRI nacional representa la imposición centrista, aunque su presidente Luis Donaldo Colosío sea Sonorense. Chávez, Elías, "Apabullados por el triunfo de Ruffo, los priístas de Baja California repudian a los enviados del centro", Proceso, (10 de julio, 1988). 
La población económicamente activa (PEA) dedicada a la agricultura es menor que el promedio nacional y la dedicada a la manufactura es mayor que el promedio nacional en todos los estados, con excepción de Sonora. La población urbana también es mayor y la población con doce años o más de educación también es superior al promedio nacional, a excepción de Chihuahua donde es ligeramente menor. Sin embargo, aun probando estas diferencias, hay que reconocer que las mismas no aportan una evidencia de que por sí mismas influyen en el comportamiento electoral.

El argumento de que la emigración genera un comportamiento electoral distinto tampoco parece sustentarse, en especial porque solamente Baja California se acerca al nivel nacional de migración interna, o sea que los habitantes son nacidos en otra entidad, los demás estados están muy por abajo del promedio nacional. Agréguese a esto que la población migra hacia la frontera norte en busca de una mejoría económica ${ }^{13}$ y no en busca de una mejoría política ${ }^{14}$.

Tratando de encontrar similitudes y diferencias, el dato sobre votación y población urbana puede ser importante para seffalar la posible socialización que implica el acto mismo de migrar. En el estudio del libro titulado Geografia de las elecciones presidenciales de México, 1988 (López, 1988) se encontró una mayor votación para la oposición en zonas urbanas. En la frontera norte la tendencia es contraria, porque, como se ve en el cuadro 4, mientras que todos los estados tienen mayor población urbana que el promedio nacional, el PRI logra más votos que en su promedio nacional en cinco de los seis estados, el PAN, en relación a su promedio nacional, logra más votos en cuatro de los estados, el PARM y el PPS solamente logran mayor votación en un estado mientras que en los otros cinco su reducción es sustancial respecto a su promedio nacional.

En el análisis de las tendencias electorales, tenemos que la abstención en general confirma la tendencia nacional que es de incremento, aunque tiene un comportamiento muy peculiar. Como se puede ver en el cuadro 5 , en algunas elecciones, en ciertos estados, el promedio es menor al

13 En 1989 el salario mínimo en Estados Unidos llegaba a \$4.25 la hora, mientras que en Mexico estaba en $\$ 8,000.00$ ( $\$ 3.33$ d6lares, calculado a 2,400 pesos por d6́lar al día). Así, el salario mexicano esta aproximadamente a .41 centavos de dolar la hora, o sea menos de una dócima parte del salario estadounidense.

14 Sin tratar de convertir en determinante una observacióng de campo, en una visita a un campo de inmigrantes mexicanos en el norte del condado de San Diego acompañando al presidente del PRI durante enero de 1990, no se not 6 que los entrevistados mostraran una eapecial preocupación por cuestiones políticas, su preocupación mayor era la relacionada con abusos, bajos salarios y malas condiciones de vida. El único argumento social que escuché fue una queja respecto a la venta de cerveza, alcohol y droga en el campo y los problemas de violencia que esto acarrea. 
CUADRO 4. Votación nacional y en seis Estados de cuatro partidos políticos y población urbana. 1988, (porcentajes)

\begin{tabular}{lrrrrrrr}
\hline & $\begin{array}{c}\text { Nacio- } \\
\text { nal }\end{array}$ & $\begin{array}{r}\text { Baja } \\
\text { Calif. }\end{array}$ & $\begin{array}{c}\text { Chihua- } \\
\text { hua }\end{array}$ & $\begin{array}{r}\text { Coa- } \\
\text { huila }\end{array}$ & $\begin{array}{r}\text { Nvo. } \\
\text { León }\end{array}$ & $\begin{array}{r}\text { Sonora Tamau- } \\
\text { lipas }\end{array}$ \\
\hline Pob. Urbana & 66.3 & 85.3 & 70.3 & 75.8 & 87.4 & 70.5 & 75.1 \\
PAN & 17.1 & 24.4 & 38.2 & 5.3 & 23.7 & 20.9 & 9.9 \\
PARM & 6.3 & 3.8 & 0.9 & 5.6 & 0.6 & 2.1 & 16.9 \\
PPS & 10.5 & 19.9 & 2.7 & 5.9 & 1.2 & 3.2 & 6.0 \\
PRI & 50.4 & 36.7 & 54.6 & 54.3 & 72.9 & 68.6 & 59.3 \\
& & & & & & & \\
\hline
\end{tabular}

FURNTE: Calculado en base a "De la trasición democrática". El Cotidiano no. 25 , sept.-oct. 1988. UAM, México.

promedio nacional para rebasarlo en la elección siguiente. La tendencia nacional registra incrementos en 1970, 1976; disminución en 1982 e incremento en 1988; solamente Tamaulipas coincide con esta tendencia; Sonora que registra el mayor índice en todo el periodo: $88.3 \%$ en 1964 , disminuye en 1970, aumenta en 1976 y 1982 y disminuye en 1988; Chihuahua aumenta en 1970 y 1976 y disminuye en 1982 y 1988; Baja California aumenta en 1970, disminuye en 1976 y 1982 y aumenta en 1988; Nuevo León disminuye en 1970, aumenta en 1976, disminuye en 1982 y aumenta en 1988 y Coahuila aumenta en 1970, disminuye en 1976 y

CUADRO 5. Abstención electoral nacional y en seis estados, 19641988, (porcentajes).

\begin{tabular}{llllllll}
\hline Año & Nal. & Tam. & Son. & Chih. & B.C. & N.L. & Coah. \\
\hline 1964 & 38.9 & 38.8 & 88.3 & 42.6 & 37.7 & 52.6 & 28.6 \\
1970 & 41.4 & 43.8 & 41.6 & 44.3 & 51.3 & 49.4 & 48.7 \\
1976 & 54.6 & 53.7 & 50.5 & 58.3 & 46.2 & 66.9 & 44.4 \\
1982 & 43.2 & 46.9 & 60.3 & 57.6 & 31.7 & 38.2 & 62.3 \\
1988 & 54.3 & 60.6 & 57.0 & 55.9 & 45.0 & 58.2 & 66.5
\end{tabular}

FUENTE: Elaborado en base a "Reforma Politica, Secretaría de Gobernación, Comisión Federal Electoral", México, 1982. "Geografía de las elecciones presidenciales de México, 1988", México, 1988. "De la transición democrática", El Cotidiano, no. 25, sept.-oct. 1988. UAM, México. 
aumenta en 1982 y 1988. Con excepción de Sonora y Chihuahua, todos los estados coinciden con el dato nacional de incremento de la abstención respecto de 1982, en 1988 solamente Coahuila tuvo un índice de abstención mayor al nacional.

Estos datos no dejan ver una tendencia muy definida pero nos muestran el comportamiento electoral; sin embargo, su dimensión puede ser distinta en virtud de que son muy frecuentes las recriminaciones de los partidos de oposición a la manipulación que el gobierno hace del padrón electoral. En términos nacionales, parece haber consenso en que el índice de error del padrón se encuentra entre el 20 y el $30 \%{ }^{15}$ pero esto no se puede probar.

En términos regionales, este autor no tiene una aproximación razonable sobre el ínđice de error en el padrón electoral. Se puede asumir que es similar al nivel nacional o se puede buscar algún otro tipo de comparación como lo hice en otro lado (Schmidt, 1988), donde encontré las discrepancias entre el padrón y la población en edad de votar. En el caso de Baja California, para 1982 el padrón electoral fue mayor a la población en edad de votar en un $8.6 \%$, y en el caso de Chihuahua el padrón electoral fue mayor a la población en edad de votar en un $9.3 \%$, por lo que tiendo a pensar que, de tener los datos exactos de población en edad de votar y de población empadronada, tendríamos un mejor acercamiento a la abstención real, luego entonces podríamos aproximarnos más al entendimiento de este fenómeno tan importante.

La votación por el PRI disminuye al igual que en la tendencia nacional, mientras que la votación por la oposición y la abstención aumenta. Aun cuando la aparición del cardenismo, representado por Cuauhtémoc Cárdenas, modificó sustancialmente las tendencias en el sentido que en $1988 \mathrm{los}$ partidos políticos menores aumentaron estrepitosamente su votación, las tres tendencias básicas se mantuvieron. Aquí de nuevo el patrón fronterizo es similar al nacional.

Como se ve en el cuadro 6, la votación en un plazo de 24 años para el PRI registra una caída constante, tanto a nivel nacional como en todos los estados fronterizos, pero solamente se reduce en mayor cantidad que en el promedio nacional consistentemente en Baja California y en Chihuahua, ya que lo hace en cuatro de las cinco elecciones reportadas; en Sonora y Tamaulipas su votación es consistentemente superior a su promedio nacional y en Nuevo León y Coahuila su votación es superior al promedio nacional en las últimas tres elecciones:

15. Dato conseguido en entrevistas del autor con funcionarios del PRI y de la Secretaría de Gobemación. 
CUADRO 6. Votación presidencial del PRI, nacional y en seis estados, 1964-1988, (porcentajes).

\begin{tabular}{llllllll}
\hline Año & Nac. & B.C. & Chih. & Coah. & N.L. & Son. & Tam. \\
\hline 1964 & 87.8 & 78.6 & 78.6 & 83.9 & 83.9 & 92.2 & 95.8 \\
1970 & 83.3 & 72.1 & 79.7 & 82.7 & 82.7 & 92.6 & 90.9 \\
1976 & 82.3 & 85.0 & 83.1 & 85.1 & 85.1 & 96.6 & 90.0 \\
1982 & 65.0 & 51.0 & 60.3 & 72.0 & 72.0 & 74.1 & 74.9 \\
1988 & 50.4 & 36.7 & 54.6 & 54.3 & 72.9 & 68.6 & 59.3 \\
\hline
\end{tabular}

FUENTE: Elaborado en base a "Reforma política, Secretaría de Gobernación, Comisión Federal Electoral", México, 1982. Gómez Tagle, Silvia, "Nos ganó la Democracia", El Cotidiano, sept-oct. 1988.

En el caso del PPS, como se ve en el cuadro 7, solamente en Baja California recibe consistentemente porcentajes de votación mayores a su promedio nacional, en los demás estados, aún en 1988, es consistentemente inferior y sólo esporádicamente es un poco superior a su promedio nacional con la sola excepción de Sonora en 1964.

El PARM, como se ve en el cuadro 8, en un plazo de 24 affos solamente en Tamaulipas recibe más votación que en el promedio nacional, en los demás estados la diferencia llega a ser significativa; pero si consideramos las dos últimas elecciones presidenciales, solamente en Sonora, Baja California y Coahuila su votación crece más de lo que lo hace en su promedio nacional.

CUADRO 7. Votación presidencial del PPS, nacional y en seis estados, 1964-1988, (porcentajes).

\begin{tabular}{lrrrrrrr}
\hline Año & Nac. & B.C. & Chih. & Coah. & N.L & .Son. & Tam. \\
\hline 1964 & 0.7 & n.d. & 0.5 & 0.2 & 0.3 & 6.2 & 0.6 \\
1970 & 0.9 & & 0.9 & 0.3 & 0.4 & 1.1 & 0.3 \\
1976 & 3.7 & 4.5 & 4.5 & 1.7 & 1.3 & 2.3 & 1.4 \\
1982 & 1.5 & 2.3 & 1.2 & & 0.5 & & 0.8 \\
1988 & 10.5 & 19.9 & 2.7 & 5.9 & 1.2 & 3.2 & 6.0 \\
\hline
\end{tabular}

FUENTE: Elaborado en base a: "Reforma política, Secretaría de Gobernación, Comisión Federal Electoral", México, 1982. Gómez Tagle, Silvia, "Nos ganó la democracia", El Cotidiano, (sept-oct. 1988). 
CUADRO 8. Votación presidencial PARM, nacional y seis estados, 1964-1988, (porcentajes).

\begin{tabular}{lccccccc}
\hline Afro & Nac. & B.C. & Chih. & Coah. & N.L. & Son. & Tam. \\
\hline 1964 & 0.5 & n.d. & 0.3 & 0.1 & 0.2 & 0.0 & 0.2 \\
1970 & 0.5 & 0.6 & 0.3 & 0.1 & 0.6 & 0.2 & 0.3 \\
1976 & 3.1 & 1.8 & 1.6 & 1.4 & 1.3 & 1.0 & 4.6 \\
1982 & 1.5 & 0.7 & 0.5 & 0.4 & 0.5 & 0.2 & 7.3 \\
1988 & 6.3 & 1.9 & 0.4 & 2.1 & 0.3 & 1.0 & 7.1 \\
\hline
\end{tabular}

FUENTE: Elaborado en base a: "Reforma política, Secretaria de Gobernación, Comisión Federal Electoral", México, 1982. Gómez Tagle, Silvia, "Nos ganó la Democracia", El Cotidiano, sept-oct. 1988.

En el caso del PAN (ver cuadro 9), su votación coincide con la tendencia de su promedio nacional, ya que en todos los estados aumenta constantemente a excepción de Baja California, Nuevo León y Coahuila que la ven disminuir en 1988. La votación en Tamaulipas es sistemáticamente inferior al promedio nacional al igual que en Coahuila en 1964, 1970 y 1988; Sonora y Chihuahua en 1964 y 1970; en el resto de los casos la votación llega a ser significativamente superior al promedio nacional, sin embargo, algo que llama la atención es que en Baja California, después de haber terminado en tercer lugar en 1988, con un decremento respecto a su

CUADRO 9. Votación presidencial PAN, nacional y seis estados, 1964-1988, (porcentajes).

\begin{tabular}{lccccccc}
\hline Año & Nac. & B.C. & Coah. & Chih. & N. L. & Son. & Tam. \\
\hline 1964 & 10.9 & 21.4 & 6.6 & 20.7 & 15.8 & 1.5 & 3.4 \\
1970 & 13.8 & 25.3 & 8.8 & 18.9 & 15.7 & 6.5 & 8.4 \\
$1976^{*}$ & 15.7 & 27.6 & 25.7 & 25.6 & 24.3 & 19.8 & 9.8 \\
1982 & 17.1 & 24.3 & 15.3 & 38.2 & 23.7 & 20.9 & 9.9 \\
1988 & & & & & & & \\
\hline
\end{tabular}

FUENTE: Elaborado en base a: "Reforma política, Secretaría de Gobernación, Comisión Federal Electoral", México, 1982. Gómez Tagle, Silvia, "Nos ganó la Democracia", El Cotidiano, (sept-oct. 1988).

* No presentó candidato presidencial. 
votación en 1982, en 1989 logre dar un gran paso y ganar para un partido opositor, por primera vez en el siglo XX, las elecciones para gobernador.

En todos los casos, y fuera de algunas de las variaciones mencionadas, la tendencia de votación es similar a la nacional sin que haya algún signo de que la frontera registra ninguna variación sustancial.

\section{LAS TENDENCIAS}

Tres tendencias son muy claras en el periodo analizado, como se ve en el cuadro 10, el PRI pierde votos sistemáticamente, el PAN gana y la abstención aumenta. Los partidos considerados "títeres del PRI", como son el PARM y PPS, tienen un comportamiento errático, que dados los bajos indices de votación que tradicionalmente reciben, llegan a registrar incrementos espectaculares.

El incremento del PFCRN en 1982 se puede explicar en base a la confusión que crearon al presentarse como el partido de Cárdenas, y es muy posible que la votación de los partidos miembros del FDN se deba en gran medida a la candidatura de Cuauhtémoc Cárdenas y no a que tengan gran cantidad de seguidores o a que le hayan quitado votos al PRI. Para ver

\section{CUADRO 10. Tasas de crecimiento de votación y abstención en elecciones presidenciales. 1964-1988. porcentajes (1964=100).}

\begin{tabular}{llrlllllr}
\hline & PAN & PARM & PFCRN & PMS & PPS & PRI & PRT & Abst. \\
\hline 1970 & 26.6 & 0.0 & & & -28.5 & -5.1 & & -6.4 \\
1976 & (a) & 520.0 & & 311.1 & -1.2 & & -31.9 \\
1982 & 13.8 & -51.6 & & & -59.5 & -21.0 & & -20.9 \\
1988 & 8.7 & 318.0 & 650.7 & 2 & 602.0 & -22.5 & -76.6 & -46.3
\end{tabular}

FUENTE: De 1964 a 1982, Schmidt, Samuel, "Votación y elecciones en la frontera México-Estados Unidos, 1961-1982". En: Schmidt, S., J.W.,Wilkie, M., Esparza, M. (compiladores), "Estudios Cuantitativos sobre la historia de México", (México, UNAM, 1988). Para 1988, Molinar Horcasitas, Juan, "Crónica del día en que el sistema se cayó-calló", El Cotidiano, no. 25, México, 1988.

(a) No presentó candidato.

NOTA: En este cuadro se incluyen solamente partidos que registran dos elecciones consecutivas, luego entonces se excluyen el PSD y el PDM. El año de comparación para PFCRN, PMS y PRT es 1982. 
si se confirma la tendencia de incremento en la votación recibida por estos partidos habrá que esperar a las elecciones de 1991 y de 1994.

\section{UN VISTAZO A LAS ELECCIONES DE 1988 Y DESPUÉS}

Bien se puede decir que las elecciones de 1988 despertaron gran interés, emoción, buenos deseos y hasta sueños. Las interpretaciones sobre las mismas están empezando a surgir y son tan optimistas o pesimistas como lo sea la orientación política de quienes las emiten, pero destaca aquélla que dice que México registró en 1988 una demanda democrática. Debo agregar que este tipo de opinión se asocia a la versión que considera que si el PRI pierde votos el país se acerca a la democracia.

Ante este tipo de opinión vale la pena mencionar que:

1. Si se suman los votos de Salinas con los Cárdenas excluyendo los del PMS, y considerando que este último abandonó al PRI mas no a su ideología, las posturas básicas del priísmo ganaron el 77.7\% de los votos, porcentaje mayor al que recibió ese partido en 1982. Esto tal vez refuerce la opinión de los comentaristas que sostienen que si Salinas no hubiera sido el candidato, dado que se le asociaba con la crisis económica, el PRI no hubiera sufrido una derrota tan fuerte.

2. Como se mencionó antes, el país vio surgir una gran cantidad de partidos políticos en todos los ámbitos del espectro ideológico y sin embargo, la abstención aumentó. Esto puede sugerir que el concepto democracia no ha penetrado a toda la sociedad y que en 1988 solamente se distribuyeron los votos que existían en el mercado electoral.

En una conversación con Manuel Clouthier (candidato del PAN a la presidencia de la república en 1988), él sostenía que el fraude electoral de 1988 consistió en una extracción de 5 millones de votos emitidos a favor del PAN para adjudicárselos al PRI, mientras que los votos computados a favor de Cuauhtémoc Cárdenas fueron los que éste realmente recibió. Si esto es así, no hay duda que las tendencias mencionadas aquí deben tener una modificación sustancial. Sin embargo, Santiago Onate, Secretario de Divulgación Ideológica del PRI en 1990, mencionó que tales cinco millones de votos representan la cantidad de votos que Clouthier pensaba que podía recibir pero que no los recibió. Lo más dramático del caso consiste en que este tipo de polémica difícilmente podrá aclararse y bien puede representar un cuadro de explicación radicalmente distinto al existente sobre el comportamiento político nacional.

Si la cuestión electoral en México implica, como parece implicar, distribución de votos existentes e inclusive un retiro proporcional creciente 
de votantes, y si las tendencias electorales en las elecciones mexicanas no han cambiado, como se demostró más arriba, diffcilmente se puede hablar de una demanda democrática.

Lo anterior no quiere decir que en México la política esté estancada. La situación político-electoral de México estácambiando a gran velocidad. El país está pasando de una situación de monopolio de un solo partido a una situación de poder compartido. El proceso de ajuste está en marcha y es difícil predecir hacia dónde se dirige o cómo estará configurado en el futuro.

Pero como es común encontrar en México, también en las elecciones hay resultados paradojicos. La información disponible permitiría suponer que las tendencias se mantendrán, esto es, el PRI seguirá reduciéndo sus votos, la abstención se mantendrá en un nivel elevado y la votación por la oposición aumentará, aunque es difícil sostener a favor de qué partido. Sin embargo, con excepción de Baja California donde por primera vez en la historia posrrevolucionaria del país un partido de oposición, el PAN, en 1989 ganó la gubernatura, la mayoría en el congreso local y la mitad de las presidencias municipales; en las 14 elecciones que se realizaron en el país durante 1989 (ver figura 2) los resultados electorales respecto a las elecciones de 1988 muestran que el PRI aumentó sus yotos en el $85.7 \%$ de los estados, el PAN aumentó su votación en el $28.6 \%$ de los estados y el PRD disminuyó sus votos en el $100 \%$ de los casos.

Los políticos no mencionan los datos sobre abstención, aunque éstos parecen ser notoriamente mayores: en Durango el 75\% (Excélsior, 13 de julio 1989), en Campeche $68.6 \%$ y en Baja California de 64 a $66 \%$ (Excélsior, 11 de julio 1989); y tampoco mencionan los hechos de violencia $^{16}$ que supuestamente no deberian existir en una país donde se avanza hacia la democracia. Ni siquiera el supuesto cambio que se nota en la cultura política (Guillén, 1989) demuestra un cambio sustancial en las tendencias electorales, aunque acaso este cambio demuestre una actitud más agresiva por parte de los miembros de los partidos de oposición o el que sectores de la población se vean sometidos a más encuestas, las que responden de forma bastante dudosa (Campuzano, 1989).

Mucho se habla también de lo volátil, oportunista y caudillesco del voto mexicano, lo que parece confirmarse en las elecciones de 1988-1989. Se puede sugerir que Cárdenas por sí mismo atrajo una gran cantidad de los votos y que en 1989 el ajuste en la coalición del FDN distribuyó los votos

16 A partir de las elecciones de 1988 la violencia electoral se ha recrudecido llegando a registrar decenas de muertos, Miller, Marjorie, "Mexico opposition party alleges 56 political killings", Los Angeles Times, january 30, 1990 y Vera, Rodrigo, "De ingobernable Michoacán paso a ingobemado", Proceso, no. 691, enero 25, 1990. 
en vías de un acomodo distinto al de 1988. En Baja California parece ser que Ernesto Ruffo logró atraer por sí mismo suficientes votos como para ganar la elección ${ }^{17}$, o que si acaso hubo manipulación de los votos ésta pudo haber sucedido en 1988 en contra del PAN o en 1989 a su favor. $\mathrm{Si}$ esto es cierto tal vez no lo sabremos, pero cualquiera que sea el tipo de manipulación, si ésta existio, arroja conclusiones políticas distintas.

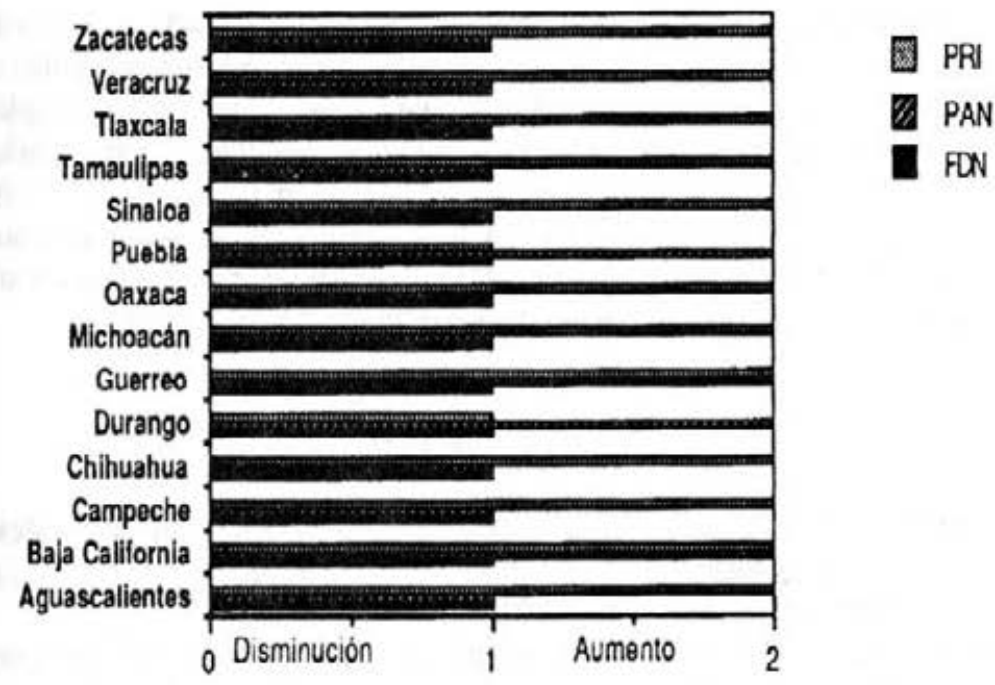

FIGURA 2. Votación en 1989. Comparación a la votación recibida en 1988.

\section{CONCLUSIÓN}

Todavía queda la impresión de que las cifras electorales se manipulan en México, y esto provoca que los estudios sobre comportamiento electoral o bien se basen en información con un elevado índice de error, por decir lo menos, o bien que sean estudios especulativos o abiertamente partidistas. Si aceptamos que la verdad ha sido manipulada y que en cierta medida es una mentira, también podemos considerar, hipotéticamente, que las

17 En estados del norte del país como Tamaulipas con frecuencia se veía en el pasado que un candidato no propuesto por el PRI podía pasarse al PARM y ganaba las elecciones, así que el argumento de voto "caudillesco" u orientado hacia el candidato y no hacia el partido tampoco es nuevo. 
tendencias mantienen una distorsión constante y por lo tanto observar las tendencias se convierte en el análisis de la verdad.

La abstención sigue siendo la opción electoral mayoritaria de los mexicanos, $y$ aun cuando alguien mencionaba que el gobiemo inflo las cifras de la abstención para perjudicar a la oposición, la información disponible no logra validar tal aserto y las tendencias en cambio le dan credibilidad al dicho de que la abstención es la opción preferida por los mexicanos.

Por último, ninguno de los partidos políticos ha logrado vencer a la abstención, pero tampoco parece que hacia futuro el sistema político electoral vaya a poder hacerlo, con lo cual México se puede contemplar como un país que tendrá una amplia oferta político-ideológica, posibilidades democráticas en el sentido de libertad de opción, pero alto rechazo de la sociedad hacia ese mismo sistema, luego entonces, por más pasos que dé el gobierno y los políticos de todos los partidos, México no se acercará a la democracia, si por ésta entendemos el gobierno del pueblo.

\section{BIBLIOGRAFÍA}

CAMPUZANO, Imma. 1989. "Una novedad: las encuestas preelectorales" en: González Fraf, Jaime (comp.). Las elecciones de 1988 y las crisis del sistema polttico. Diana-IMEP, México

DÁVILA, Enrique. 1987. "Plataformas electorales e fndice analítico", en: Meyenberg, Yolanda, et al. Polttica y partidos en las elecciones federales de 1985. México, UNAM. Facultad de Ciencias Sociales y Políticas.

DOWNS, Anthony. 1973. Teorla económica de la democracia. Ed. Aguilar, España.

EQUIPO DE COYUNTURA. 1988. "De la transición democrática”. El Cotidiano, no. 25, UAM-I, México.

GÓMEZ Tagle, Silvia. 1988. "Nos ganó la democracia". El Cotidiano. sep.-oct. 1988.

GUILLEN, Tonatiúh. 1989. "La cultura política y la elección presidencial de 1988. Hacia un análisis del neocardenismo". Frontera Norte, no.1 enero-julio 1989.

LÓPEZ, Arturo. 1988. Geografla de las elecciones presidenciales de México, 1988. Fundación Arturo Rosenblueth, México.

MOLINAR Horcasitas, Juan. 1988. "Crónicas del día en que el sistema se cayó-callo". El Cotidiano, no. 25, UAM-I.

PAOLI, José. 1979. "Primer fracaso de la reforma política". Proceso, no. 142. 
PEACH, James T. 1984. "Demographic and economic change in Mexico's northern frontier. Evidence from the $x$ Censo General de Poblacion y Vivienda". Las Cruces, New Mexico State University, Center for Latin American Studies (Latin America Research Papers, no. 3). SCHMIDT, Samuel. 1988. "Votación y elecciones en la frontera MéxicoEstados Unidos. 1961-1982”. En: Schmidt, S., Wilkie, J., Esparza, M. (Comp.). Estudios cuantitativos sobre la historia de México. México, UNAM.

SECRETARÍA DE GOBERNACIÓN. 1982. Reforma política, Comisión Federal Electoral, México.

WILKIE, J. y D. Lory (eds.). 1987. Statistical abstract of Latin America. Vol. 23, Los Angeles, UCLA: Latin American Center Publications. 